\title{
RESULTS FROM ASCA SKY SURVEYS
}

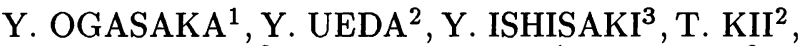 \\ T. TAKAHASHI ${ }^{2}$, K. MAKISHIMA $^{4}, \mathrm{H}_{\text {. INOUE }}{ }^{2}, \mathrm{~K}^{2} \mathrm{OHTA}^{5}$, \\ T. YAMADA ${ }^{6}$, T. MIYAJI $^{7}$ AND G. HASINGER ${ }^{8}$ \\ ${ }^{1}$ NASA Goddard Space Flight Center \\ ${ }^{2}$ The Institute of Space and Astronautical Science \\ ${ }^{3}$ Department of Physics, Tokyo Metropolitan University \\ ${ }^{4}$ Department of Physics, University of Tokyo \\ ${ }^{5}$ Department of Astronomy, Kyoto University \\ ${ }^{6}$ Institute of Physical and Chemical Research (RIKEN) \\ ${ }^{7}$ Max-Planck-Institute für Extraterrestrische Physik \\ ${ }^{8}$ Astrophysikalisches Institut Potsdam
}

\section{Introduction}

The origin of the Cosmic X-ray Background (CXB) radiation has been investigated extensively by soft X-ray deep survey imaging observations with Einstein and ROSAT. In contrast, the lack of telescopes capable of detecting hard $\mathrm{X}$-rays has prevented us from extensive study of the nature of the CXB in the energy range above $2 \mathrm{keV}$ before $A S C A$.

$A S C A$ Deep Sky Survey (DSS) and Large Sky Survey (LSS) were intended to carry out unbiased surveys in the wide energy range of $0.5-10$ $\mathrm{keV}$. DSS was planned to survey a small sky region with extremely high sensitivity reaching to the source confusion limit of the $A S C A$ XRT, while LSS covers a much larger sky area with relatively shallow exposures. These two surveys play complimentary roles in our approaches to the nature of the faint X-ray objects and the origin of the CXB, especially in the 2-10 $\mathrm{keV}$ band.

\section{DSS Results}

The DSS consists of moderately deep pointings of five sky regions and extremely deep pointings of Selected Area 57 (SA57) (Ogasaka 1996). The data reduction and analysis have been done for the Lynx Field, the Lockman Hole and a part of SA57, covering a total solid angle of $0.29 \mathrm{deg}^{2}$. The $N(>S)$ in the $2-10 \mathrm{keV}$ band was derived as $55 \pm 25 \mathrm{deg}^{-2}$ at the flux limit 
of $3.80 \times 10^{-14} \mathrm{erg} \mathrm{sec}^{-1} \mathrm{~cm}^{-2}$. This is consistent with the extrapolation of $\log N-\log S$ relations from previous experiments(e.g., Hayashida 1991; Piccinotti et al. 1982). At this flux limit about $40 \%$ of the CXB intensity in the $2-10 \mathrm{keV}$ band is resolved into discrete sources. On the other hand, the $N(>S)$ in the $0.5-2 \mathrm{keV}$ band is consistent with more sensitive ROSAT $\log N-\log S$ relation derived by Hasinger et al. (1993).

\section{LSS Results}

The Large Sky Survey, where a continuous sky regions near the North Galactic Pole is systematically surveyed, covered $6 \mathrm{deg}^{2}$ up to the present (Ueda 1996). We detected $\sim 50$ sources in the survey energy band of 2-10 $\mathrm{keV}$ whose flux distribute from $1.5 \times 10^{-13}$ to $2.0 \times 10^{-12} \mathrm{erg} \mathrm{sec}^{-1} \mathrm{~cm}^{-2}$. The derived $\log N-\log S$ relation above $2 \mathrm{keV}$ is consistent with the extrapolation from the previous results. The average spectrum for the sources with flux less than $2.5 \times 10^{-13} \mathrm{erg} \mathrm{sec}^{-1} \mathrm{~cm}^{-2}$ shows a photon index of $1.5 \pm 0.2$ above $2 \mathrm{keV}$, which is harder than that of bright AGN observed so far. This result suggests that the hard sources responsible for the CXB above $2 \mathrm{keV}$ appear when the sensitivity drops to $\sim 10^{-13} \mathrm{erg} \mathrm{sec}^{-1} \mathrm{~cm}^{-2}$.

\section{Source Identifications}

We have carried out the optical follow-up observations for X-ray sources detected from the Lynx, SA57 and LSS fields. Significant fraction of hard $\mathrm{X}$-ray dominated sources were identified with narrow-line objects. One of them is the narrow-line quasar at $\mathrm{z} \simeq 0.9$ discovered from the Lynx Field (Ohta et al. 1996). It is possible that this object is the "type-2 quasar," whose existence has been expected from the unified model of AGNs, and from the "spectral paradox" of the CXB.

\section{Acknowledgements}

YO acknowledges the support of the Postdoctoral Fellowships for Research Abroad of the Japan Society for the Promotion of the Science.

\section{References}

Hasinger, G. et al., 1993, Astron.Astrophys., 275, 1.

Hayashida, K. 1989, Ph. D dissertation of Univ. of Tokyo, ISAS RN 466.

Ogasaka, Y. 1996, Ph. D dissertation of Gakushuin University

Ohta, K. et al., 1996, Astrophys.J., 458, L57

Piccinotti, G. et al. 1982, Astrophys.J., 253, 485.

Ueda, Y. 1996, Ph. D dissertation of University of Tokyo 